\title{
USE OF SEMIDEFINITE PROGRAMMING FOR SOLVING THE LQR PROBLEM SUBJECT TO RECTANGULAR DESCRIPTOR SYSTEMS
}

\author{
MUHAFZAN \\ Department of Mathematics \\ Andalas University, Kampus Unand Limau Manis Padang, 25163 Indonesia \\ e-mail: muhafzan@gmail.com
}

\begin{abstract}
This paper deals with the Linear Quadratic Regulator (LQR) problem subject to descriptor systems for which the semidefinite programming approach is used as a solution. We propose a new sufficient condition in terms of primal dual semidefinite programming for the existence of the optimal state-control pair of the problem considered. The results show that semidefinite programming is an elegant method to solve the problem under consideration. Numerical examples are given to illustrate the results.
\end{abstract}

Keywords: rectangular descriptor system, LQR problem, restricted system equivalent, semidefinite programming.

\section{Introduction}

The semidefinite programming problem is an optimization one in which the decision variables are semidefinite matrices. It is well known that semidefinite programming has been one of the most exciting and active research areas in optimization recently. This tremendous activity is spurred by the discovery of important applications in various areas, mainly in control theory (Balakrishnan and Vandenberghe, 2003; Rami and Zhou, 2000; Vandenberghe and Boyd, 1999).

In the work of Yao et al. (2001), semidefinite programming was used to find optimal control of the classical LQR (linear quadratic regulator) problem. It is well known that this problem has many applications, mainly in electrical engineering, biology, social sciences and economics.

On the other hand, the LQR problem subject to a descriptor system is a generalization of the the classical LQR problem, which is one of the most important classes of optimal control problems, in both theory and application. In general, it is a problem to find a controller that minimizes the linear quadratic objective function subject to the descriptor systems, either continuous or discrete.

Descriptor systems have attracted the attention of many researcher in the past years due to the fact that, in some cases, they describe the behavior of physical systems better than standard systems do. They can preserve the structure of physical systems and include a nondy- namic constraint and an impulsive element. Systems of this kind have many important applications, e.g., in biological phenomena, in economics (the Leontief dynamic model), in electrical and mechanical models (Dai, 1989; Silva and de Lima, 2003). Therefore, it is fair to say that descriptor systems give a more complete class of dynamical models than conventional state-space systems do. Likewise, the LQR problem subject to descriptor systems has great potential for system modelling.

A great number of results on solving the LQR problem subject to descriptor systems have appeared in the literature (Bender and Laub, 1987; Geerts, 1994; Jiandong et al., 2002; Katayama and Minamino, 1992; Mehrmann, 1989). However, almost all of these results consider the regularity assumption of descriptor systems and the positive definiteness assumption of the control weighting matrix in the quadratic cost functional.

To the best of the author's knowledge, little work has been done with rectangular descriptor systems as a constraint and the control weighting matrix in the quadratic cost being positive semidefinite. In this last case, i.e., the quadratic cost being positive semidefinite, the existing LQR problem theories always involve impulse distributions (Geerts, 1994; Mehrmann, 1989). Thus no answer is provided to a basic question such as when the LQR problem subject to descriptor systems possesses an optimal solution in the form of a conventional control, in particular, one that does not involve an impulse distribution. However, this issue was discussed by Jiandong et al. (2002), 
who transform the LQR problem subject to a descriptor system into a standard LQR problem in which both are equivalent. Nonetheless, there still remains an open problem, that is, the new standard LQR problem may be singular and this is not answered by Jiandong et al. (2002).

In this paper, we reconsider the problem stated by Jiandong et al. (2002) and, in particular, the open problem, i.e., the singular version of the new standard LQR problem, is solved using the semidefinite programming approach. A new sufficient condition in terms of primal dual semidefinite programming for the existence of the optimal state-control pair of such a problem is proposed. Here, since we do not assume that the descriptor system is regular, our work is more general than some previous results (Bender and Laub, 1987; Geerts, 1994; Katayama and Minamino, 1992; Mehrmann, 1989). The method of Jiandong et al. (2002) is still maintained to transform the original problem into the equivalent singular LQR one.

This paper is organized as follows. Section 2 considers a brief account of the problem statement. Section 3 presents the process of transformation from the original $\mathrm{LQR}$ problem into an equivalent $\mathrm{LQR}$ one. In Section 4, the main result for solving the LQR problem subject to descriptor system is presented. Numerical examples are given to illustrate the results in Section 5. Section 6 concludes the paper.

Notation. Throughout this paper, the superscript ' $T$ ' stands for the transpose, $\varnothing$ denotes the empty set, $I_{n}$ is the identity matrix of dimension $n, \mathbb{R}^{n}$ denotes the $n$ dimensional Euclidean space, $\mathbb{R}^{m \times n}$ is the set of all $m \times n$ real matrices, $\mathbb{C}_{\mathrm{p}}^{+}\left[\mathbb{R}^{n}\right]$ denotes the $n$-dimensional piecewise continuous functions space with domain in $[0, \infty)$, $\mathbb{S}_{+}^{p}$ denotes set of all $p$-dimensional symmetric positive semidefinite matrices, and $\mathbb{C}$ denotes the set of complex number.

\section{Problem statement}

Let us consider the following continuous time descriptor system:

$$
\begin{aligned}
E \dot{x}(t) & =A x(t)+B u(t), \quad t \geq 0, \quad E x(0)=x_{0}, \\
y(t) & =C x(t)+D u(t),
\end{aligned}
$$

where $x(t) \in \mathbb{R}^{n}$ denotes the state variable, $u(t) \in$ $\mathbb{R}^{r}$ denotes the control (input) variable and $y(t) \in \mathbb{R}^{q}$ denotes the output variable. The matrices $E, A \in \mathbb{R}^{m \times n}$, $B \in \mathbb{R}^{m \times r}, C \in \mathbb{R}^{q \times n}, D \in \mathbb{R}^{q \times r}$ are constant, with $\operatorname{rank} E \equiv p<\min \{m, n\}$. This system is denoted by $(E, A, B, C, D)$. The system $(E, A, B, C, D)$ is said to be regular if $m=n$ and $\operatorname{det}(s E-A) \neq 0$ for almost all $s \in \mathbb{C}$. Otherwise, it is called nonregular if $\operatorname{det}(s E-A)=0$ for each $s \in \mathbb{C}$ or if $E, A \in \mathbb{R}^{m \times n}$ with $m \neq n$. In particular, it is called a rectangular descriptor system if $m \neq n$.
It is well known that the solution to (1) exists and is unique if this system is regular. Otherwise, it is possible to have many solutions, or no solution at all.

Next, for a given admissible initial state $x_{0} \in \mathbb{R}^{n}$, we consider the following associated objective function (cost functional):

$$
J\left(u(\cdot), x_{0}\right)=\int_{0}^{\infty} y^{T}(t) y(t) \mathrm{d} t .
$$

In general, the problem of determining the stabilizing feedback control $u(t) \in \mathbb{R}^{r}$ which minimizes the cost functional (2) on the the trajectories of the dynamic system (1) for an admissible initial state $x_{0} \in \mathbb{R}^{n}$ is often called the LQR problem subject to a descriptor system. If $D^{T} D$ is positive semidefinite, it is called a singular LQR problem subject to a descriptor system.

In this paper, we will consider the LQR problem subject to a rectangular descriptor system. Without loss of generality, we assume that $m<n$. We denote, for simplicity, this LQR problem as $\Omega$. Next, we define the set of admissible control-state pairs of the problem $\Omega$ by

$$
\begin{aligned}
& \mathbb{A}_{\mathrm{ad}} \equiv\left\{(u(\cdot), x(\cdot)) \mid u(\cdot) \in \mathbb{C}_{\mathrm{p}}^{+}\left[\mathbb{R}^{r}\right]\right. \text { and } \\
& x(\cdot) \in \mathbb{C}_{\mathrm{p}}^{+}\left[\mathbb{R}^{n}\right] \text { satisfy (1) and } \\
& \left.\qquad J\left(u(\cdot), x_{0}\right)<\infty\right\} .
\end{aligned}
$$

The problem under consideration is how to use semidefinite programming to find the pair $\left(u^{*}, x^{*}\right) \in \mathbb{A}_{\mathrm{ad}}$ for a given admissible initial condition $x_{0} \in \mathbb{R}^{n}$, such that

$$
J\left(u^{*}, x_{0}\right)=\min _{(u(\cdot), x(\cdot)) \in \mathbb{A}_{\mathrm{ad}}} J\left(u(\cdot), x_{0}\right),
$$

under the assumption that (1) is solvable, impulse controllable and $D^{T} D$ is positive semidefinite.

Definition 1. (Jiandong et al., 2002) Two systems $(E, A, B, C, D)$ and $(\bar{E}, \bar{A}, \bar{B}, \bar{C}, D)$ are termed restricted system equivalent (r.s.e.), denoted by

$$
(E, A, B, C, D) \sim(\bar{E}, \bar{A}, \bar{B}, \bar{C}, D),
$$

if there exist two nonsingular matrices $M \in \mathbb{R}^{m \times m}$ and $N \in \mathbb{R}^{n \times n}$ such that their associated system matrices are related by $M E N=\bar{E}, M A N=\bar{A}, M B=\bar{B}$ and $C N=\bar{C}$.

Definition 2. (Jiandong et al., 2002) Two optimal control problems are said to be equivalent if there exists a bijection between the two admissible control-state pair sets, and the value of the quadratic cost functional of an image is equal to that of the corresponding preimage.

Obviously, Definition 2 confirms that two equivalent optimal control problems will have the same solvability, uniqueness of solution and optimal cost characterizations. Thus solving one can be replaced by solving the other. 


\section{Transformation into an equivalent $L Q R$ problem}

Since $\operatorname{rank} E=p<m$, the Singular Value Decomposition (SVD) theorem (Klema and Laub, 1980) implies that there exist nonsingular matrices $M \in \mathbb{R}^{m \times m}$ and $N \in \mathbb{R}^{n \times n}$ such that

$$
M E N=\left(\begin{array}{cc}
I_{p} & 0 \\
0 & 0
\end{array}\right)
$$

It follows that we have

$$
\begin{gathered}
M A N=\left(\begin{array}{ll}
A_{11} & A_{12} \\
A_{21} & A_{22}
\end{array}\right), \quad M B=\left(\begin{array}{c}
B_{1} \\
B_{2}
\end{array}\right), \\
C N=\left(\begin{array}{ll}
C_{1} & C_{2}
\end{array}\right), \quad N^{-1} x=\left(\begin{array}{l}
x_{1} \\
x_{2}
\end{array}\right),
\end{gathered}
$$

where $A_{11} \in \mathbb{R}^{p \times p}, A_{12} \in \mathbb{R}^{p \times(n-p)}, A_{21} \in \mathbb{R}^{(m-p) \times p}$, $A_{22} \in \mathbb{R}^{(m-p) \times(n-p)}, B_{1} \in \mathbb{R}^{p \times r}, B_{2} \in \mathbb{R}^{(m-p) \times r}, C_{1} \in$ $\mathbb{R}^{q \times p}, C_{2} \in \mathbb{R}^{q \times(n-p)}, x_{1} \in \mathbb{R}^{p}$ and $x_{2} \in \mathbb{R}^{n-p}$. Therefore, for a given admissible initial state $x_{0} \in \mathbb{R}^{n}$, the system (1) is r.s.e. to the system

$$
\begin{aligned}
\dot{x}_{1}(t) & =A_{11} x_{1}(t)+A_{12} x_{2}(t)+B_{1} u(t), \\
0 & =A_{21} x_{1}(t)+A_{22} x_{2}(t)+B_{2} u(t), \\
y(t) & =C_{1} x_{1}(t)+C_{2} x_{2}(t)+D u(t),
\end{aligned}
$$

with $x_{1}(0)=x_{10}=\left(\begin{array}{ll}I_{p} & 0\end{array}\right) M x_{0}$.

Using the expression (5), the objective function (2) can be changed into

$$
J_{1}\left(u(\cdot), x_{10}\right)=\int_{0}^{\infty} \bar{x}^{T}(t) Q \bar{x}(t) \mathrm{d} t,
$$

where

$$
\begin{aligned}
\bar{x}(t) & =\left(\begin{array}{c}
x_{1}(t) \\
x_{2}(t) \\
u(t)
\end{array}\right), \\
Q & =\left(\begin{array}{ccc}
C_{1}^{T} C_{1} & C_{1}^{T} C_{2} & C_{1}^{T} D \\
C_{2}^{T} C_{1} & C_{2}^{T} C_{2} & C_{2}^{T} D \\
D^{T} C_{1} & D^{T} C_{2} & D^{T} D
\end{array}\right) .
\end{aligned}
$$

Likewise, we have the new LQR problem which minimizes the objective function $J_{1}\left(u(\cdot), x_{10}\right)$ subject to the dynamic system (5), and denote this LQR problem as $\Omega_{1}$. Further, we define the set of admissible control-state pairs of the problem $\Omega_{1}$ by

$$
\begin{aligned}
\mathbb{A}_{\mathrm{ad}}^{1} \equiv\left\{\left(u(\cdot), x_{1}(\cdot), x_{2}(\cdot)\right) \mid u(\cdot) \in \mathbb{C}_{\mathrm{p}}^{+}\left[\mathbb{R}^{r}\right],\right. \\
x_{1}(\cdot) \in \mathbb{C}_{\mathrm{p}}^{+}\left[\mathbb{R}^{p}\right] \text { and } x_{2}(\cdot) \in C_{\mathrm{p}}^{+}\left[\mathbb{R}^{n-p}\right] \\
\left.\quad \text { satisfy (5) and } J_{1}\left(u(\cdot), x_{10}\right)<\infty\right\} .
\end{aligned}
$$

By virtue of Definition 2, it is easily seen that the LQR problem $\Omega_{1}$ is equivalent to $\Omega$.
From the work of Ishihara and Terra (2001), we know that impulsive controllability of the descriptor system (11) is equivalent to

$$
\operatorname{rank}\left(\begin{array}{ccc}
A_{21} & A_{22} & B_{2}
\end{array}\right)=\operatorname{rank}\left(\begin{array}{ll}
A_{22} & B_{2}
\end{array}\right) .
$$

In fact, the matrix $\left(\begin{array}{cc}A_{22} & B_{2}\end{array}\right)$ may have no full row rank. Let us write $\operatorname{rank}\left(\begin{array}{cc}A_{22} & B_{2}\end{array}\right)=s$, where $s \leq m-p \leq n-p$. It follows that there exists a nonsingular matrix $V \in \mathbb{R}^{(m-p) \times(m-p)}$ such that

$$
V\left(\begin{array}{ll}
A_{22} & B_{2}
\end{array}\right)=\left(\begin{array}{cc}
\bar{A}_{22} & \bar{B}_{2} \\
0 & 0
\end{array}\right),
$$

where $\left(\begin{array}{cc}\bar{A}_{22} & \bar{B}_{2}\end{array}\right)$ has full row rank. By modifying the procedure of Jiandong et al. (2002), we have the following transformation:

$$
\bar{x}(t)=\left(\begin{array}{cc}
I_{p} & 0 \\
0 & W
\end{array}\right) \Gamma \bar{x}_{1}(t),
$$

where

$$
\Gamma=\left(\begin{array}{c|c}
I_{p} & 0 \\
\hline-\bar{A}_{21} & 0 \\
0 & I_{n-p+r-s}
\end{array}\right)
$$

and

$$
\bar{x}_{1}(t)=\left(\begin{array}{c}
x_{1}(t) \\
v(t)
\end{array}\right)
$$

for some $v \in \mathbb{R}^{n-p+r-s}$ and for some nonsingular matrix

$$
W=\left(\begin{array}{ll}
W_{11} & W_{12} \\
W_{21} & W_{22}
\end{array}\right) \in \mathbb{R}^{(n-p+r) \times(n-p+r)},
$$

where $\bar{A}_{21}=\left(\begin{array}{ll}I_{s} & 0\end{array}\right) V A_{21}$. Finally, we get a new LQR problem as follows:

$$
\begin{gathered}
\min _{\left(v(\cdot), x_{1}\right)} J_{2}\left(v(\cdot), x_{10}\right)=\int_{0}^{\infty} \bar{x}_{1}^{T}(t) \bar{Q} \bar{x}_{1}(t) d t, \\
\text { s.t. }\left\{\begin{array}{r}
\dot{x}_{1}(t)=\bar{A} x_{1}(t)+\bar{B}_{1} v(t), \quad x_{1}(0)=x_{10}, \\
y(t)=\bar{C} x_{1}(t)+\bar{D} v(t)
\end{array}\right.
\end{gathered}
$$

where

$$
\begin{aligned}
\bar{Q} & =\left(\begin{array}{ll}
Q_{11} & Q_{12} \\
Q_{12}^{T} & Q_{22}
\end{array}\right), \\
\bar{A} & =A_{11}-\bar{A}_{12} \bar{A}_{21}, \\
\bar{A}_{12} & =A_{12} W_{11}+B_{1} W_{21}, \\
\bar{B}_{1} & =A_{12} W_{12}+B_{1} W_{22}, \\
\bar{C} & =C_{1}-\left(\begin{array}{cc}
C_{2} & D
\end{array}\right) W\left(\begin{array}{c}
\bar{A}_{21} \\
0
\end{array}\right), \\
\bar{D} & =\left(\begin{array}{cc}
C_{2} & D
\end{array}\right) W\left(\begin{array}{c}
0 \\
I_{n-p+r-s}
\end{array}\right), \\
Q_{11} & =\bar{C}^{T} \bar{C}, \quad Q_{12}=\bar{C}^{T} \bar{D}, \quad Q_{22}=\bar{D}^{T} \bar{D} .
\end{aligned}
$$


Let us denote by $\Omega_{2}$, this LQR problem and define the set of admissible control-state pairs of the problem $\Omega_{2}$ by

$$
\begin{aligned}
& \mathbb{A}_{\mathrm{ad}}^{2} \equiv\left\{\left(v(\cdot), x_{1}(\cdot)\right) \mid v(\cdot) \in \mathbb{C}_{\mathrm{p}}^{+}\left[\mathbb{R}^{n-p+r-s}\right]\right. \text { and } \\
& x_{1}(\cdot) \in \mathbb{C}_{\mathrm{p}}^{+}\left[\mathbb{R}^{p}\right] \text { satisfy }(\underline{8}) \text { and } \\
& \left.\qquad J_{2}\left(v(\cdot), x_{10}\right)<\infty\right\} .
\end{aligned}
$$

It is obvious that the system (8) is a standard state space system with the state $x_{1}$, the control $v$ and the output $y$, so $\Omega_{2}$ is a standard LQR problem.

It is easy to show that the transformation defined by (7) is a bijection from $\mathbb{A}_{\text {ad }}^{2}$ to $\mathbb{A}_{\text {ad }}^{1}$, and thus the problem $\Omega_{2}$ is equivalent to the problem $\Omega_{1}$. It follows that $\Omega_{2}$ is equivalent to the problem $\Omega$ as well. Therefore, in order to solve the problem $\Omega$, it suffices to consider the problem $\Omega_{2}$ only.

\section{Solving the LQR problem}

It is well known that the solution of $\Omega_{2}$ hinges on the behavior of the input weighting matrix $Q_{22}$ in (9), whether it is positive definite or positive semidefinite.

Under a certain property, $Q_{22}$ may be positive definite, (see the results of Jiandong et al. (2002) for details). In the case where $Q_{22}$ is positive definite, one can use the classical theory of LQR, which asserts that $\Omega_{2}$ has a unique optimal control-state pair if the pair $\left(\bar{A}, \bar{B}_{1}\right)$ is asymptotically stabilizable and the pair $(\bar{A}-$ $\bar{B}_{1} Q_{22}^{-1} Q_{12}^{T}, Q_{11}-Q_{12} Q_{22}^{-1} Q_{12}^{T}$ ) is detectable (Anderson and Moore, 1990). In this case, the optimal control $v^{*}$ is given by

$$
v^{*}=L x_{1}^{*},
$$

where the state $x_{1}^{*}$ is the solution to the differential equation

$$
\dot{x}_{1}(t)=\left(\bar{A}+\bar{B}_{1} L\right) x_{1}(t), \quad x_{1}(0)=x_{10},
$$

with $L=-Q_{22}^{-1}\left(Q_{12}^{T}+\bar{B}_{1}^{T} P\right)$, and $P$ is the unique positive semidefinite solution of the following algebraic Riccati equation:

$$
\begin{aligned}
\bar{A}^{T} P & +P \bar{A}+Q_{11} \\
& -\left(P \bar{B}_{1}+Q_{12}\right) Q_{22}^{-1}\left(P \bar{B}_{1}+Q_{12}\right)^{T}=0,
\end{aligned}
$$

where every eigenvalue $\lambda$ of $\left(A_{11}-\bar{A}_{12} \bar{A}_{21}+\bar{B}_{1} L\right)$ satisfies $\operatorname{Re} \lambda<0$. Thus, in this case, the optimal controlstate pair of the problem $\Omega$ is given by

$$
\begin{aligned}
& \left(\begin{array}{l}
x^{*} \\
u^{*}
\end{array}\right) \\
& \quad=\left(\begin{array}{cc}
N & 0 \\
0 & I_{r}
\end{array}\right)\left(\frac{-W_{11} \bar{A}_{21}+W_{12} L}{-W_{21} \bar{A}_{21}+W_{22} L}\right) x_{1}^{*} .
\end{aligned}
$$

On the other hand, when the matrix $Q_{22}$ is positive semidefinite $\left(Q_{22} \geq 0\right)$, the algebraic Riccati equation (12) seems to be meaningless, and therefore this result can no longer be used to handle the singular LQR problem $\Omega$.

A natural extension is to generalize the algebraic Riccati equation (12) by replacing the matrix $Q_{22}^{-1}$ with the matrix $Q_{22}^{\dagger}$, such that the equation (12) is replaced by

$$
\begin{aligned}
\digamma(P) & \equiv \bar{A}^{T} P+P \bar{A}+Q_{11} \\
& -\left(P \bar{B}_{1}+Q_{12}\right) Q_{22}^{\dagger}\left(P \bar{B}_{1}+Q_{12}\right)^{T}=0,
\end{aligned}
$$

where $Q_{22}^{\dagger}$ stands for the Moore-Penrose inverse of $Q_{22}$. Consider an affine transformation of the matrix $P$ as follows:

$$
\mathcal{H}(P) \equiv\left(\begin{array}{cc}
Q_{22} & \left(P \bar{B}_{1}+Q_{12}\right)^{T} \\
P \bar{B}_{1}+Q_{12} & Q_{11}+\bar{A}^{T} P+P \bar{A}
\end{array}\right) .
$$

By using Schur's Extended lemma (Rami and Zhou, 2000), we have the following lemma, which shows that $\digamma(P) \geq 0$ and $\mathcal{H}(P) \geq 0$ are closely related.

Lemma 1. (Balakrishnan and Vandenberghe, 2003) $\left(I_{n-p+r-s}-Q_{22} Q_{22}^{\dagger}\right)\left(P \bar{B}_{1}+Q_{12}\right)^{T}=0$ and $\digamma(P) \geq$ 0 if and only if $\mathcal{H}(P) \geq 0$.

Now, let us consider the following primal semidefinite programming:

$$
\begin{array}{|cc|}
\hline \max & \left\langle I_{p}, P\right\rangle, \\
\text { s.t. } & P \in \mathcal{P}, \\
\hline
\end{array}
$$

where

$$
\mathcal{P} \equiv\left\{P \in \mathbb{S}_{+}^{p} \mid \mathcal{H}(P) \geq 0\right\}
$$

is the set of feasible solutions of the primal semidefinite programming problem $(\mathrm{P})$. It is easy to show that $\mathcal{P}$ is a convex set and it may be empty which in particular implies that there is no solution to primal semidefinite programming. Moreover, it is easy to show that the objective function of the problem $(\mathrm{P})$ is convex as well. Since the objective function and $\mathcal{P}$ satisfy the convexity properties, the above primal semidefinite programming case is a convex optimization problem.

Corresponding to the above primal semidefinite programming, we have the following dual problem:

$$
\begin{gathered}
\min \left\langle Q_{22}, Z_{b}\right\rangle+2\left\langle Q_{12}^{T}, Z_{u}\right\rangle+\left\langle Q_{11}, Z_{p}\right\rangle, \\
\text { s.t. } Z_{u}^{T} \bar{B}_{1}^{T}+\bar{B}_{1} Z_{u}+Z_{p} \bar{A}^{T}+\bar{A} Z_{p}+I_{p}=0, \\
Z \equiv\left[\begin{array}{cc}
Z_{b} & Z_{u} \\
Z_{u}^{T} & Z_{p}
\end{array}\right] \geq 0
\end{gathered}
$$

where $Z$ denotes the dual variable associated with the primal constraint $\mathcal{H}(P) \geq 0$ with $Z_{b}, Z_{u}$ and $Z_{p}$ being a block partitioning of $Z$ of appropriate dimensions. 
Remark 1. Semidefinite programming is known to be a special form of conic optimization problems, for which there exists a well-developed duality theory (see, e.g., the works of Balakrishnan and Vandenberghe (2003), Rami and Zhou (2000), Vandenberghe and Boyd (1999) or Yao et al. (2001) for an exhaustive theory of semidefinite programming). Key points of the theory can be highlighted as follows:

1. Weak duality always holds, i.e., any feasible solution to the primal problem always possesses an objective value that is greater than the dual objective value of any dual feasible solution. In contrast, strong duality does not always hold.

2. A sufficient condition for strong duality is that there exist a pair of complementary optimal solution, i.e., both the primal and dual semidefinite programming problems have attainable optimal solutions, and that these solutions are complementary to each other. This means that the optimal solution $P^{*}$ and the dual optimal solution $Z^{*}$ both exist and satisfy $\mathcal{H}\left(P^{*}\right) Z^{*}=0$.

3. If both (P) and (D) satisfy strict feasibility, namely, there exist a primal and dual feasible solution $P_{0}$ and $Z_{0}$ such that $\mathcal{H}\left(P_{0}\right)>0$ and $Z_{0}>0$, then the complementary solutions exist.

In the following we present the condition for the stability of the singular LQR control problem $\Omega_{2}$.

Theorem 1. The singular LQR problem $\Omega_{2}$ is stabilizable if and only if the dual problem (D) is strictly feasible.

Proof. $\quad(\Rightarrow)$ First assume that the system (8) is stabilizable by some feedback control $v(t)=L x_{1}(t)$. Then all the eigenvalues of the matrix $\bar{A}+\bar{B}_{1} L$ have negative real parts. Consequently, using Lyapunov's theorem (Balakrishnan and Vandenberghe, 2003), there exists a positive definite matrix $Y$ such that

$$
\left(\bar{A}+\bar{B}_{1} L\right) Y+Y\left(\bar{A}+\bar{B}_{1} L\right)^{T}=-I_{p} .
$$

By setting $Z_{p}=Y$ and $Z_{u}=L Z_{p}$, this relation can be rewritten as

$$
Z_{u}^{T} \bar{B}_{1}^{T}+\bar{B}_{1} Z_{u}+Z_{p} \bar{A}^{T}+\bar{A} Z_{p}+I_{p}=0 .
$$

Now choose

$$
Z_{b}=\epsilon I_{r}+Z_{u}\left(Z_{p}\right)^{-1} Z_{u}^{T}
$$

Then, by Schur's lemma, $Z$ is strictly feasible to (D).

$(\Leftarrow)$ If the dual problem (D) is strictly feasible, then $Z_{p}>$ 0 by Schur's lemma. Setting

$$
L=Z_{u}\left(Z_{p}\right)^{-1},
$$

with $Z$ satisfying the equality constraint of (D) yields

$$
\left(\bar{A}+\bar{B}_{1} L\right) Z_{p}+Z_{p}\left(\bar{A}+\bar{B}_{1} L\right)^{T}=-I_{p} .
$$

By constructing a quadratic Lyapunov function $x_{1}^{T} Z_{p} x_{1}$, it is easily verified that the system in (8) is stabilizable.

Theorem 2. If (P) and (D) satisfy the complementary slackness condition, then the optimal solution of the problem $(\mathrm{P})$ satisfies the generalized algebraic Riccati equation $\digamma(P)=0$.

Proof. Let $P^{*}$ and $Z^{*}$ denote the optimal solution of $(\mathrm{P})$ and (D), respectively. Since $P^{*}$ is optimal, it is also feasible and satisfies $\mathcal{H}\left(P^{*}\right) \geq 0$. By Lemma 1 , we have

$$
\left(I_{r}-Q_{22} Q_{22}^{\dagger}\right)\left(P^{*} \bar{B}_{1}+Q_{12}\right)^{T}=0 .
$$

Thus, the following decomposition is true:

$$
\begin{aligned}
\mathcal{H}\left(P^{*}\right)= & \left(\begin{array}{cc}
I_{r} & 0 \\
\left(P^{*} \bar{B}_{1}+Q_{12}\right) Q_{22}^{\dagger} & I_{p}
\end{array}\right) \\
& \times\left(\begin{array}{cc}
Q_{22} & 0 \\
0 & \digamma\left(P^{*}\right)
\end{array}\right) \\
& \times\left(\begin{array}{cc}
I_{r} & Q_{22}^{\dagger}\left(P^{*} \bar{B}_{1}+Q_{12}\right)^{T} \\
0 & I_{p}
\end{array}\right) .
\end{aligned}
$$

From the relation $\mathcal{H}\left(P^{*}\right) Z^{*}=0$, we have

$$
\mathcal{H}\left(P^{*}\right) Z^{*}=\left(\begin{array}{ll}
\mathcal{H}_{11} & \mathcal{H}_{12} \\
\mathcal{H}_{21} & \mathcal{H}_{22}
\end{array}\right)=\left(\begin{array}{ll}
0 & 0 \\
0 & 0
\end{array}\right),
$$

where

$$
\begin{aligned}
& \mathcal{H}_{11}=Q_{22}\left(Z_{b}^{*}+Q_{22}^{\dagger}\left(P^{*} \bar{B}_{1}+Q_{12}\right)^{T}\left(Z_{u}^{*}\right)^{T}\right), \\
& \mathcal{H}_{12}=Q_{22}\left(Z_{u}^{*}+Q_{22}^{\dagger}\left(P^{*} \bar{B}_{1}+Q_{12}\right)^{T} Z_{p}^{*}\right), \\
& \mathcal{H}_{21}=\digamma\left(P^{*}\right)\left(Z_{u}^{*}\right)^{T}, \\
& \mathcal{H}_{22}=\digamma\left(P^{*}\right) Z_{p}^{*} .
\end{aligned}
$$

Therefore

$$
\digamma\left(P^{*}\right)\left(Z_{u}^{*}\right)^{T}=0 \text { and } \digamma\left(P^{*}\right) Z_{p}^{*}=0,
$$

and hence

$$
Z_{u}^{*} \digamma\left(P^{*}\right)=0 \text { and } Z_{p}^{*} \digamma\left(P^{*}\right)=0 .
$$

Since $Z^{*}$ is dual feasible, it also satisfies

$$
Z_{u}^{T} \bar{B}_{1}^{T}+\bar{B}_{1} Z_{u}+Z_{p} \bar{A}^{T}+\bar{A} Z_{p}+I_{p}=0 .
$$

Pre-and post-multiplying the above equation by $\digamma\left(P^{*}\right)$, we get,

$$
\digamma\left(P^{*}\right)\left(Z_{u}^{T} \bar{B}_{1}^{T}+\bar{B}_{1} Z_{u}+Z_{p} \bar{A}^{T}+\bar{A} Z_{p}+I_{p}\right) \digamma\left(P^{*}\right),
$$

which yields $\digamma\left(P^{*}\right)^{2}=0$ and hence $\digamma\left(P^{*}\right)=0$. 
Now, let us consider the subset $\mathcal{P}_{\text {bound }}$ of $\mathcal{P}$ which is defined as follows:

$$
\mathcal{P}_{\text {bound }} \equiv\left\{P \in \mathbb{S}_{+}^{p} \mid \mathcal{H}(P) \geq 0 \text { and } \digamma(P)=0\right\} .
$$

Note that $\mathcal{P}_{\text {bound }}$ may be empty, which in particular implies that there is no solution to the generalized algebraic Riccati equation (14).

In the following, we present our main results, where the LQR problem is explicitly constructed in terms of the solution to primal and dual semidefinite programming.

Theorem 3. If $\mathcal{P}_{\text {bound }} \neq \varnothing$ and

$$
v^{*}(t)=-Q_{22}^{\dagger}\left(P^{*} \bar{B}_{1}+Q_{12}\right)^{T} x_{1}(t)
$$

is a stabilizing control for some $P^{*} \in \mathcal{P}_{\text {bound, where }}$ $x_{1}(t)$ satisfies the differential equation

$$
\dot{x}_{1}(t)=\left(\bar{A}-\bar{B}_{1} Q_{22}^{\dagger}\left(P^{*} \bar{B}_{1}+Q_{12}\right)^{T}\right) x_{1}(t),
$$

with $x_{1}(0)=x_{10}$, then $(\mathrm{P})$ and $(\mathrm{D})$ satisfy the complementary slackness property. Moreover, $v^{*}(t)$ is the optimal control for the LQR problem $\Omega_{2}$.

Proof. $\quad$ Let $P^{*} \in \mathcal{P}_{\text {bound }}$ and $L=-Q_{22}^{\dagger}\left(P^{*} \bar{B}_{1}+\right.$ $\left.Q_{12}\right)^{T}$. Since the control $v^{*}(t)=L x_{1}(t)$ is stabilizing, the Lyapunov equation

$$
\left(\bar{A}+\bar{B}_{1} L\right) Y+Y\left(\bar{A}+\bar{B}_{1} L\right)^{T}+I_{p}=0
$$

has a positive definite solution. Denote it be $Y^{*}>0$. Let

$$
Z_{p}^{*}=Y^{*}, \quad Z_{u}^{*}=L Y^{*}, Z_{b}^{*}=L Y^{*} L^{T} .
$$

By this construction, we can easily verify that

$$
\begin{aligned}
& \left(\begin{array}{cc}
Z_{b}^{*} & Z_{u}^{*} \\
\left(Z_{u}^{*}\right)^{T} & Z_{p}^{*}
\end{array}\right) \\
& \quad=\left(\begin{array}{cc}
I_{r} & L \\
0 & I_{p}
\end{array}\right)\left(\begin{array}{cc}
0 & 0 \\
0 & Z_{p}^{*}
\end{array}\right)\left(\begin{array}{cc}
I_{r} & 0 \\
L^{T} & I_{p}
\end{array}\right) \\
& \quad \geq 0
\end{aligned}
$$

and

$$
I_{p}+\left(Z_{u}^{*}\right)^{T} \bar{B}_{1}^{T}+\bar{B}_{1} Z_{u}^{*}+Z_{p}^{*} \bar{A}^{T}+\bar{A} Z_{p}^{*}=0
$$

Therefore,

$$
Z^{*}=\left(\begin{array}{cc}
Z_{b}^{*} & Z_{u}^{*} \\
\left(Z_{u}^{*}\right)^{T} & Z_{p}^{*}
\end{array}\right)
$$

is a feasible solution of (D). Since $\mathcal{H}(P) \geq 0$, by Lemma 1, we have

$$
\left(I_{r}-Q_{22} Q_{22}^{\dagger}\right)\left(P \bar{B}_{1}+Q_{12}\right)^{T}=0 .
$$

It follows that the identity

$$
\begin{aligned}
\mathcal{H}(P)= & \left(\begin{array}{cc}
I_{r} & 0 \\
\left(P \bar{B}_{1}+Q_{12}\right) Q_{22}^{\dagger} & I_{p}
\end{array}\right) \\
& \times\left(\begin{array}{cc}
Q_{22} & 0 \\
0 & \digamma(P)
\end{array}\right) \\
& \times\left(\begin{array}{cc}
I_{r} & Q_{22}^{\dagger}\left(P \bar{B}_{1}+Q_{12}\right)^{T} \\
0 & I_{p}
\end{array}\right)
\end{aligned}
$$

is valid. Moreover, we can verify that

$$
\begin{aligned}
\mathcal{H} & \left(P^{*}\right) Z^{*} \\
= & \left(\begin{array}{cc}
I_{r} & 0 \\
-L^{T} & I_{p}
\end{array}\right)\left(\begin{array}{cc}
Q_{22} & 0 \\
0 & \digamma\left(P^{*}\right)
\end{array}\right) \\
& \times\left(\begin{array}{cc}
I_{r} & -L \\
0 & I_{p}
\end{array}\right)\left(\begin{array}{cc}
Z_{b}^{*} & Z_{u}^{*} \\
\left(Z_{u}^{*}\right)^{T} & Z_{p}^{*}
\end{array}\right) \\
= & \left(\begin{array}{cc}
I_{r} & 0 \\
-L^{T} & I_{p}
\end{array}\right) \\
& \times\left(\begin{array}{cc}
Q_{22}\left(Z_{b}^{*}-L\left(Z_{u}^{*}\right)^{T}\right) & Q_{22}\left(Z_{u}^{*}-L Z_{p}^{*}\right) \\
\digamma & \left.\digamma P^{*}\right)\left(Z_{u}^{*}\right)^{T}
\end{array}\right) \\
= & \left(\begin{array}{cc}
0 & 0 \\
0 & 0
\end{array}\right),
\end{aligned}
$$

that is, Problems (P) and (D) satisfy the complementary slackness property. Now, we prove that

$$
v^{*}(t)=-Q_{22}^{\dagger}\left(P^{*} \bar{B}_{1}+Q_{12}\right)^{T} x_{1}(t)
$$

is the optimal control for the LQR problem $\Omega_{2}$. Firstly, consider any $P \in \mathcal{P}$ and any admissible stabilizing control $v(\cdot) \in \mathbb{C}_{\mathrm{p}}^{+}\left[\mathbb{R}^{r}\right]$. We have

$$
\begin{aligned}
\frac{\mathrm{d}}{\mathrm{d} t}( & \left.x_{1}^{T}(t) P x_{1}(t)\right) \\
= & \left(\bar{A} x_{1}(t)+\bar{B}_{1} v(t)\right)^{T} P x_{1}(t) \\
& +x_{1}^{T}(t) P\left(\bar{A} x_{1}(t)+\bar{B}_{1} v(t)\right) \\
= & x_{1}^{T}(t)\left(\bar{A}^{T} P+P \bar{A}\right) x_{1}(t)+2 v^{T}(t) \bar{B}_{1}^{T} P x_{1}(t) .
\end{aligned}
$$

Integrating over $[0, \infty)$ and making use of the fact that

$$
\lim _{t \rightarrow \infty} x_{1}^{T}(t) P x_{1}(t)=0
$$

we have

$$
\begin{aligned}
0= & x_{10}^{T} P x_{10}+\int_{0}^{\infty}\left(x_{1}^{T}(t)\left(\bar{A}^{T} P+P \bar{A}\right) x_{1}(t)\right. \\
& \left.+2 v^{T}(t) \bar{B}_{1}^{T} P x_{1}(t)\right) \mathrm{d} t .
\end{aligned}
$$


Therefore,

$$
\begin{aligned}
J_{2} & \left(v(\cdot), x_{10}\right) \\
= & \int_{0}^{\infty}\left(x_{1}^{T}(t) Q_{11} x_{1}(t)\right. \\
& \left.+2 v^{T}(t) Q_{12}^{T} x_{1}(t)+v^{T}(t) Q_{22} v(t)\right) \mathrm{d} t \\
= & x_{10}^{T} P x_{10}+\int_{0}^{\infty}\left(x_{1}^{T}(t)\left(\bar{A}^{T} P+P \bar{A}+Q_{11}\right) x_{1}(t)\right. \\
& \left.+2 v^{T}(t)\left(P \bar{B}_{1}+Q_{12}\right)^{T} x_{1}(t)+v^{T}(t) Q_{22} v(t)\right) \mathrm{d} t \\
= & x_{10}^{T} P x_{10} \\
& +\int_{0}^{\infty}\left[\left(v(t)+Q_{22}^{+}\left(P \bar{B}_{1}+Q_{12}\right)^{T} x_{1}(t)\right)^{T} Q_{22}\right. \\
& \times\left(v(t)+Q_{22}^{+}\left(P \bar{B}_{1}+Q_{12}\right)^{T} x_{1}(t)\right) \\
& \left.+x_{1}^{T}(t) \digamma(P) x_{1}(t)\right] \mathrm{d} t .
\end{aligned}
$$

Since $P \in \mathcal{P}$, we have $\digamma(P) \geq 0$. This means that

$$
J_{2}\left(v(\cdot), x_{10}\right) \geq x_{10}^{T} P x_{10},
$$

for each $P \in \mathcal{P}$ and each admissible stabilizing control $v(\cdot) \in \mathbb{C}_{\mathrm{p}}^{+}\left[\mathbb{R}^{r}\right]$. On the other hand, under the feedback control

$$
v^{*}(t)=-Q_{22}^{\dagger}\left(P^{*} \bar{B}_{1}+Q_{12}\right)^{T} x_{1}(t),
$$

and if we take into account $P^{*} \in \mathcal{P}_{\text {bound }}$, we have

$$
\begin{aligned}
0 \leq & J_{2}\left(v^{*}(\cdot), x_{10}\right) \\
= & \int_{0}^{\infty}\left[x_{1}^{T}(t) Q_{11} x_{1}(t)+2 v^{*^{T}}(t) Q_{12}^{T} x_{1}(t)\right. \\
& \left.+v^{*^{T}}(t) Q_{22} v^{*}(t)\right] \mathrm{d} t \\
= & \lim _{t \rightarrow \infty} \int_{0}^{t}\left[x_{1}^{T}(\tau) Q_{11} x_{1}(\tau)+2 v^{*^{T}}(\tau) Q_{12}^{T} x_{1}(\tau)\right. \\
& \left.+v^{*^{T}}(\tau) Q_{22} v^{*}(\tau)\right] \mathrm{d} \tau \\
= & \lim _{t \rightarrow \infty}\left[x_{10}^{T} P^{*} x_{10}-x_{1}^{T}(t) P^{*} x_{1}(t)\right. \\
& +\int_{0}^{t}\left(x_{1}^{T}(\tau)\left(\bar{A}^{T} P^{*}+P^{*} \bar{A}+Q_{11}\right) x_{1}(\tau)\right. \\
& +2 v^{*^{T}}(\tau)\left(P^{*} \bar{B}_{1}+Q_{12}\right)^{T} x_{1}(\tau) \\
& \left.\left.+v^{*^{T}}(\tau) Q_{22} v^{*}(\tau)\right) \mathrm{d} \tau\right] \\
\leq & x_{10}^{T} P^{*} x_{10}+\lim _{t \rightarrow \infty} \int_{0}^{t}\left[\left(v^{*}(\tau)\right.\right. \\
& \left.+Q_{22}^{\dagger}\left(P^{*} \bar{B}_{1}+Q_{12}\right)^{T} x_{1}(\tau)\right)^{T} Q_{22} \\
& \times\left(v^{*}(\tau)+Q_{22}^{\dagger}\left(P^{*} \bar{B}_{1}+Q_{12}\right)^{T} x_{1}(\tau)\right) \\
& \left.+x_{1}^{T}(\tau) \digamma\left(P^{*}\right) x_{1}(\tau)\right] \mathrm{d} \tau \\
= & x_{10}^{T} P^{*} x_{10} .
\end{aligned}
$$

It follows that

$$
J_{2}\left(v^{*}(\cdot), x_{10}\right) \leq x_{10}^{T} P^{*} x_{10} .
$$

The facts (17) and (18) lead us to conclude that the LQR problem $\Omega_{2}$ has an attainable optimal feedback control which is given by (16) with the cost $x_{10}^{T} P^{*} x_{10}$.

The significance of Theorem 3 is that one can solve the LQR problem for a standard state space system by simply solving a corresponding semidefinte programming problem. Consequently, since there exists the equivalent relationship between the $\mathrm{LQR}$ problem subject to rectangular descriptor systems and the standard LQR problem, one can also solve the $\mathrm{LQR}$ problem subject to rectangular descriptor systems via such the corresponding semidefinite programming approach.

By reconsidering the transformation (7), it follows that

$$
\begin{aligned}
\left(\begin{array}{l}
x_{1}^{*}(t) \\
x_{2}^{*}(t) \\
u^{*}(t)
\end{array}\right) & =\left(\begin{array}{cc}
I_{p} & 0 \\
0 & W
\end{array}\right) \Gamma\left(\begin{array}{l}
x_{1}^{*}(t) \\
v^{*}(t)
\end{array}\right) \\
& =\left(\begin{array}{cc}
I_{p} & 0 \\
-W_{11} \bar{A}_{21} & W_{12} \\
-W_{21} \bar{A}_{21} & W_{22}
\end{array}\right)\left(\begin{array}{c}
x_{1}^{*}(t) \\
v^{*}(t)
\end{array}\right) \\
& =\left(\begin{array}{c}
I_{p} \\
\Lambda_{1} \\
\Lambda_{2}
\end{array}\right) x_{1}^{*}(t),
\end{aligned}
$$

where $\Lambda_{1}=-W_{11} \bar{A}_{21}-W_{12} Q_{22}^{\dagger}\left(P^{*} \bar{B}_{1}+Q_{12}\right)^{T}$ and $\Lambda_{2}=-W_{21} \bar{A}_{21}-W_{22} Q_{22}^{\dagger}\left(P^{*} \bar{B}_{1}+Q_{12}\right)^{T}$. Finally, by reconsidering (4), we obtain that the optimal control-state pair $\left(u^{*}, x^{*}\right)$ of $\Omega$ is

$$
x^{*}=N\left(\begin{array}{c}
I_{p} \\
-W_{11} \bar{A}_{21}-W_{12} Q_{22}^{\dagger}\left(P^{*} \bar{B}_{1}+Q_{12}\right)^{T}
\end{array}\right) x_{1}^{*}
$$

and

$$
u^{*}=\left(-W_{21} \bar{A}_{21}-W_{22} Q_{22}^{\dagger}\left(P^{*} \bar{B}_{1}+Q_{12}\right)^{T} x_{1}^{*} .\right.
$$

We end this section by presenting a sufficient condition for the existence of the optimal control of the $\mathrm{LQR}$ problem subject to a descriptor system.

Corollary 1. Assume that the rectangular descriptor system (17) is impulse controllable and the $\mathrm{LQR}$ problem $\Omega$ is equivalent to $\Omega_{2}$, where the matrix $Q_{22}$ is positive semidefinite. If $\mathcal{P}_{\text {bound }} \neq \varnothing$ and

$$
v^{*}(t)=-Q_{22}^{\dagger}\left(P^{*} \bar{B}_{1}+Q_{12}\right)^{T} x_{1}(t)
$$

is a stabilizing control for some $P^{*} \in \mathcal{P}_{\text {bound, }}$, where $x_{1}(t)$ satisfies the differential equation

$$
\dot{x}_{1}(t)=\left(\bar{A}-\bar{B}_{1} Q_{22}^{\dagger}\left(P^{*} \bar{B}_{1}+Q_{12}\right)^{T}\right) x_{1}(t),
$$


with $x_{1}(0)=x_{10}$, then

$u^{*}(t)=\left(-W_{21} \bar{A}_{21}-W_{22} Q_{22}^{\dagger}\left(P^{*} \bar{B}_{1}+Q_{12}\right)^{T}\right) x_{1}(t)$

is the optimal control for the LQR problem subject to the rectangular descriptor system (11).

\section{Numerical example}

Consider the LQR problem subject to the rectangular descriptor system, where the matrices $E, A, B, C$ and $D$ are given as follows:

$$
\begin{aligned}
E & =\left(\begin{array}{lllll}
1 & 0 & 0 & 0 & 0 \\
0 & 1 & 0 & 0 & 0 \\
0 & 0 & 0 & 0 & 0 \\
0 & 0 & 0 & 0 & 0
\end{array}\right), \\
A & =\left(\begin{array}{rrrrr}
-3 & 6 & 1 & 0 & 0 \\
-1 & -4 & -1 & -1 & 3 \\
0 & -2 & 0 & -1 & 2 \\
0 & -2 & 0 & -1 & 2
\end{array}\right), \\
B & =\left(\begin{array}{rr}
1 & -1 \\
1 & 2 \\
1 & 0 \\
1 & 0
\end{array}\right), \quad C=\left(\begin{array}{lllll}
1 & 0 & 0 & 0 & 0 \\
0 & 0 & 0 & 0 & 0
\end{array}\right), \\
D & =\left(\begin{array}{cc}
-1 & -1 \\
0 & 0
\end{array}\right),
\end{aligned}
$$

with the initial state

$$
x_{0}=\left(\begin{array}{cccc}
0 & 1 & 0 & 0
\end{array}\right)^{T} .
$$

By taking the matrices $M=I_{4}$ and $N=I_{5}$, it is easy to verify that

$$
\operatorname{rank}\left(\begin{array}{lll}
A_{21} & A_{22} & B_{2}
\end{array}\right)=\operatorname{rank}\left(\begin{array}{ll}
A_{22} & B_{2}
\end{array}\right)=1,
$$

and thus the rectangular descriptor system is impulse controllable. By choosing

$$
V=\left(\begin{array}{cc}
0.5 & 0.5 \\
0.7071 & 0.7071
\end{array}\right)
$$

and

$$
W=\left(\begin{array}{rrrrr}
0 & 0 & 0 & -1 & 0 \\
2 & 3 & 0 & 2 & 1 \\
1 & 1 & -1 & 1 & 0 \\
1 & 1 & 2 & 0 & 1 \\
0 & 0 & 0 & 0 & -1
\end{array}\right),
$$

the problem $\Omega$ can be equivalently changed into the singular LQR problem subject to the standard state space system:

$$
\begin{aligned}
& \min _{\left(v(.), x_{1}\right)} \int_{0}^{\infty}\left(\begin{array}{c}
x_{1}(t) \\
v(t)
\end{array}\right)^{T} \bar{Q}\left(\begin{array}{c}
x_{1}(t) \\
v(t)
\end{array}\right) \mathrm{d} t, \\
& \text { s.t. }\left\{\begin{array}{l}
\dot{x}_{1}(t)=\bar{A} x_{1}(t)+\bar{B}_{1} v(t), \quad x_{1}(0)=\left(\begin{array}{l}
0 \\
1
\end{array}\right) \\
y(t)=\bar{C} x_{1}(t)+\bar{D} v(t),
\end{array}\right.
\end{aligned}
$$

where $x_{1} \in \mathbb{R}^{2}, v \in \mathbb{R}^{4}$,

$$
\begin{aligned}
& \bar{A}=\left(\begin{array}{ll}
-3 & 8 \\
-1 & 0
\end{array}\right), \quad \bar{B}_{1}=\left(\begin{array}{cccc}
1 & 2 & -1 & 2 \\
1 & -1 & 2 & -2
\end{array}\right), \\
& \bar{C}=\left(\begin{array}{cc}
1 & -2 \\
0 & 0
\end{array}\right), \quad \bar{D}=\left(\begin{array}{rrrr}
-1 & -2 & 0 & 0 \\
0 & 0 & 0 & 0
\end{array}\right) \text {, } \\
& Q_{11}=\left(\begin{array}{cc}
1 & -2 \\
-2 & 4
\end{array}\right), \quad Q_{12}=\left(\begin{array}{rrrr}
-1 & -2 & 0 & 0 \\
2 & 4 & 0 & 0
\end{array}\right) \text {, }
\end{aligned}
$$

and

$$
Q_{22}=\left(\begin{array}{cccc}
1 & 2 & 0 & 0 \\
2 & 4 & 0 & 0 \\
0 & 0 & 0 & 0 \\
0 & 0 & 0 & 0
\end{array}\right)
$$

To identify a positive semidefinite feasible solution $P^{*}$ to primal semidefinite programming that satisfies the generalized algebraic Riccati equation $\digamma\left(P^{*}\right)=0$, we first consider the constraint

$$
\left(I_{4}-Q_{22} Q_{22}^{\dagger}\right)\left(P^{*} \bar{B}_{1}+Q_{12}\right)^{T}=0
$$

as stipulated by Lemma 1, such that we have

$$
\left(\begin{array}{cc}
1.2 q & 1.2 r \\
-0.6 q & -0.6 r \\
-p+2 q & -q+2 r \\
2 p-2 q & 2 q-2 r
\end{array}\right)=\left(\begin{array}{cc}
0 & 0 \\
0 & 0 \\
0 & 0 \\
0 & 0
\end{array}\right)
$$

This is satisfied only by $p=q=r=0$, i.e.,

$$
P^{*}=\left(\begin{array}{ll}
0 & 0 \\
0 & 0
\end{array}\right) \text {. }
$$

In fact, this $P^{*}$ satisfies the generalized algebraic Riccati equation (14). It follows that

$$
\bar{A}-\bar{B}_{1} Q_{22}^{\dagger}\left(P^{*} \bar{B}_{1}+Q_{12}\right)^{T}=\left(\begin{array}{cc}
-2 & 6 \\
-1.2 & 0.4
\end{array}\right),
$$

which has eigenvalues $-0.8+2.4 i$ and $-0.8-2.4 i$, and these are stable. Hence the control

$$
v^{*}(t)=-Q_{22}^{\dagger}\left(P^{*} \bar{B}_{1}+Q_{12}\right)^{T} x_{1}^{*}(t),
$$

where $x_{1}^{*}(t)$ is solution of the following differential equation

$$
\dot{x}_{1}(t)=\left(\begin{array}{cc}
-2 & 6 \\
-1.2 & 0.4
\end{array}\right) x_{1}(t), \quad x_{1}(0)=\left(\begin{array}{l}
0 \\
1
\end{array}\right),
$$

is stabilizing. It is easy to verify that

$$
x_{1}^{*}(t)=e^{-0.8 t}\left(\begin{array}{c}
2.5 \sin 2.4 t \\
0.5(2 \cos 2.4 t+\sin 2.4 t)
\end{array}\right) .
$$

Hence, according to Theorem 3, the control $v^{*}(t)$ must be optimal to the singular $\mathrm{LQR}$ problem $\Omega_{2}$. 


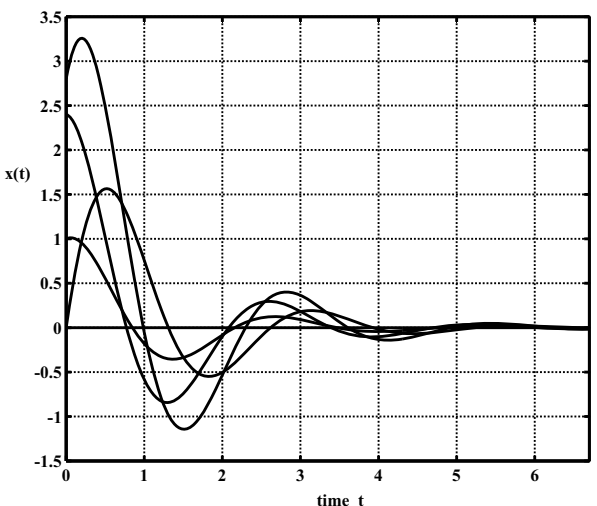

(a)

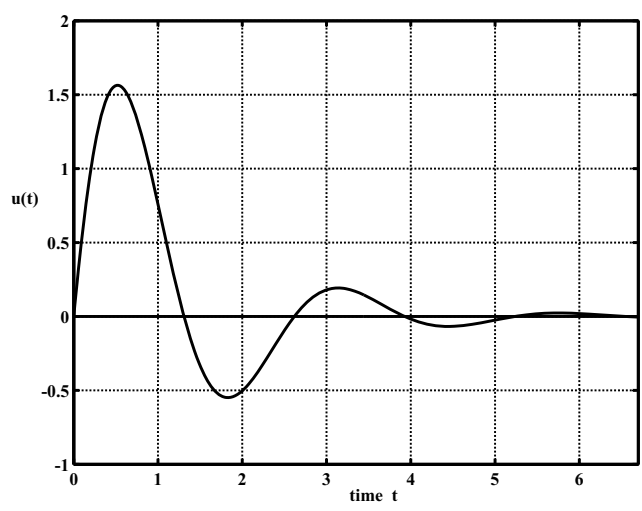

(b)

Fig. 1. Simulation results: state trajectories (a), control trajectories (b).

Thereby, according to Corollary 1, the optimal statecontrols are as follows:

$$
\begin{aligned}
& x^{*}(t)=e^{-0.8 t}\left(\begin{array}{c}
2.5 \sin 2.4 t \\
0.5(2 \cos 2.4 t+\sin 2.4 t) \\
0 \\
0.1(28 \cos 2.4 t+29 \sin 2.4 t) \\
0.1(24 \cos 2.4 t+7 \sin 2.4 t)
\end{array}\right), \\
& u^{*}(t)=\left(\begin{array}{c}
2.5 e^{-0.8 t} \sin 2.4 t \\
0
\end{array}\right),
\end{aligned}
$$

and the optimal cost $J_{\mathrm{opt}}=0$.

The trajectories of the state $x$ and the control $u$ are shown in Fig. 1.

\section{Conclusion}

We have solved the LQR control problem subject to a rectangular descriptor system using the semidefinite programming approach. We have also proposed a new sufficient condition in terms of semidefinite programming for the existence of the optimal state-control pair of the problem considered. The results show that the optimal control-state pair is free impulse, i.e., they are smooth functions.

\section{Acknowledgment}

The author would like to thank the anonymous referees for very helpful suggestions and comments which improved the quality of this paper. This work was supported by the Ministry of Education of Indonesia through Grant No. 437/SP2H/PP/DP2M/V/2009.

\section{References}

Anderson, B.D.O. and Moore, J.B. (1990). Optimal Control: Linear Quadratic Methods, Prentice-Hall, Upper Saddle River, NJ.
Balakrishnan, V. and Vandenberghe, L. (2003). Semidefinite programming duality and linear time-invariant systems, IEEE Transactions on Automatic Control 48(1): 30-41.

Bender, D.J. and Laub, A.J. (1987). The linear quadratic optimal regulator for descriptor systems, IEEE Transactions on Automatic Control 32(8): 672-688.

Dai, L. (1989). Singular Control Systems, Lecture Notes in Control and Information Sciences, Vol. 118, Springer, Berlin.

Geerts, T. (1994). Linear quadratic control with and without stability subject to general implicit continuous time systems: Coordinate-free interpretations of the optimal cost in terms of dissipation inequality and linear matrix inequality, Linear Algebra and Its Applications 203-204: 607-658.

Ishihara, J.Y. and Terra, M.H. (2001). Impulse controllability and observability of rectangular descriptor systems, IEEE Transactions on Automatic Control 46: 991-994.

Jiandong, Z., Shuping, M. and Zhaolin, C. (2002). Singular LQ problem for nonregular descriptor system, IEEE Transactions on Automatic Control 47(7): 1128-1133.

Katayama, T. and Minamino, K. (1992). Linear quadratic regulator and spectral factorization for continuous time descriptor system, Proceedings of the IEEE Conference on Decision and Control, Tucson, AZ, USA, pp. 967-972.

Klema, V.C. and Laub, A.J. (1980). The singular value decomposition: Its computation and some applications IEEE Transactions on Automatic Control 25(2): 164-176.

Mehrmann, V. (1989). Existence, uniqueness, and stability of solutions to singular linear quadratic optimal control problems, Linear Algebra and Its Applications 121: 291-331.

Rami, M.A. and Zhou, X.Y. (2000). Linear matrix inequalities, riccati equations, and indefinite stochastic linear quadratic controls, IEEE Transactions on Automatic Control 45(6): 1131-1143.

Silva, M.S. and de Lima, T.P. (2003). Looking for nonnegative solutions of a leontif dynamic model, Linear Algebra and Its Applications 364: 281-316. 
Vandenberghe, L. and Boyd, S. (1999). Applications of semidefinite programming, Applied Numerical Mathematics 29: 283-299.

Yao, D., Zhang, D. and Zhou, X.Y. (2001). A primal dual semidefinite programming approach to linear quadratic control, IEEE Transactions on Automatic Control 46(9): 1442-1447.

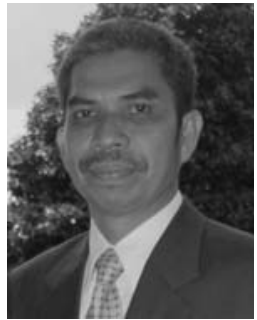

Muhafzan received the B.Sc. degree in mathematics from Riau University (Indonesia) in 1992, the M.Sc. degree in mathematics from the Bandung Institute of Technology (Indonesia) in 1999, and the $\mathrm{Ph} . \mathrm{D}$ degree in applied mathematics from Universiti Putra Malaysia in 2007. He has been a lecturer at Andalas University, Indonesia, since 1993. His research interests include the theory of descriptor systems and optimal control.

Received: 13 August 2009

Revised: 15 February 2010

Re-revised: 24 June 2010 